\begin{tabular}{|c|l|}
\hline Title & Theoretical and numerical investigations on grain boundary migration due to inverse pinning \\
\hline Author(s) & Ohno, Munekazu; Ohyama, Shinpei; Matsuura, Kiyotaka \\
\hline Citation & $\begin{array}{l}\text { Computational materials science, 79, 558.563 } \\
\text { https://doi.org/40.1016/.commatsci.2013.07.007 }\end{array}$ \\
\hline Issue Date & 201311 \\
\hline Doc URL & http://hdl.handle.net/2115/53664 \\
\hline Type & article \\
\hline File Information & 20130hno_inversepinning_text_01.pdf \\
\hline
\end{tabular}

Instructions for use 


\title{
Theoretical and numerical investigations on grain boundary migration due to inverse pinning
}

\author{
Munekazu Ohno ${ }^{\text {a }}$, Shinpei Ohyama ${ }^{\mathrm{b}}$ and Kiyotaka Matsuura ${ }^{\mathrm{a}}$ \\ ${ }^{a}$ Division of Materials Science and Engineering, Faculty of Engineering, Hokkaido University, Kita \\ 13 Nishi 8, Kita-ku, Sapporo, Hokkaido 060-8628, Japan \\ ${ }^{b}$ Graduate School of Engineering, Hokkaido University
}

Corresponding author: Dr. Munekazu Ohno

Faculty of Engineering, Hokkaido University

Kita 13 Nishi 8, Kita-ku, Sapporo, 060-8628, Japan

Tel: +81-(0)11-706-6344

Fax: +81-(0)11-706-6344

E-mail address: mohno@eng.hokudai.ac.jp

\begin{abstract}
:
Theoretical and numerical investigations are carried out into the inverse pinning effect of platelet particles on the grain boundary migration. The theoretical expression for the driving force of the inverse pinning proposed in an early work is first revisited and modified to remove the limitation in the volume fraction of the particles. In addition, it is shown that there exists the maximum velocity of the grain boundary due to the inverse pinning. The validity of the present considerations is demonstrated by phase-field simulations for the grain growth.
\end{abstract}

Keywords; Grain growth; Inverse pinning; Phase-field simulation 


\section{Introduction}

Utilization of second phase particles in controlling grain structures of polycrystalline materials is one of the important techniques, the essential ingredient of which is the physics of the particle-boundaries interaction. It has been widely acknowledged that the existence of the particles causes retardation of the grain boundary migration during the grain growth processes. There have been a number of theoretical, experimental and simulation studies on this aspect, i.e., the pinning effect [1-10]. On the other hand, as exemplified by the case of AlN particles in steels [11], the particles with the anisotropic interfacial energy can induce and accelerate the migration of grain boundaries. Nishizawa called this phenomenon "inverse pinning" [11].

In contrast to the usual pinning effect, little has been clarified for the inverse pinning effect. It is often the case that interface properties between the matrix and second phase particle exhibit a strong anisotropy and, in this respect, the inverse pinning effect is considered as one of the effects often emerging in material production processes. This effect was recently investigated by phase-field simulations for the grain growth and it was found that it is highly possible that the inverse pinning causes the abnormal grain growth [12]. Hence, it is important to elucidate the detail of the inverse pinning effect, especially its kinetics.

Nishizawa proposed that the driving force for the inverse pinning of platelet particles $\Delta G_{i n v}\left(\mathrm{~J} \mathrm{~m}^{-3}\right)$ can be approximated by the following equation [11].

$$
\Delta G_{\text {inv }}=\frac{2 v_{\mathrm{m}} f_{\mathrm{v}}}{w_{\mathrm{p}}}\left(\sigma_{2}-\sigma_{1}\right)
$$

where $v_{\mathrm{m}}$ is the molar volume of the matrix, $f_{\mathrm{v}}$ is the volume fraction of the particle, $w_{\mathrm{p}}$ is the thickness of the platelet particles and $\sigma_{1}\left(\sigma_{2}\right)$ is the interfacial energy between the particle and grain 1 (grain 2). When $\sigma_{2}>\sigma_{1}$, the boundary migrates due to $\Delta G_{\text {inv }}$, yielding the growth of grain 1 and the shrink of grain 2 . It should be pointed out that the detailed exposition was not given for the derivation of Eq. (1) in Ref. [11] and several important points remain to be analyzed and elucidated especially regarding the validity and application range of this equation. Although the validity of this relation was demonstrated for some cases by phase-field simulations [12], a more detailed and systematic investigation is required for deeper understanding of this effect and its practical utilization in material production processes. As will be shown later, Eq. (1) is validated for the system with small volume fraction of platelet particles. Therefore, it is not applicable to systems with relatively high volume fraction of the particles.

In this paper, Eq. (1) is first re-derived and modified to eliminate the limitation of the small volume fraction. Then, the kinetics of the inverse pinning is analyzed, 
which elucidates the maximum velocity of the grain boundary moving due to the inverse pinning. Finally, the validity of these discussions is investigated by phase-field simulations.

\section{Driving force of inverse pinning}

The driving force of the inverse pinning $\Delta G_{\text {inv }}$ given by Eq. (1) is first reexamined. $\Delta G_{\mathrm{inv}}$ is now expressed as $\Delta G_{\mathrm{inv}}=v_{\mathrm{m}} \Delta P_{\mathrm{inv}}$ with the inverse pinning pressure $\Delta P_{\text {inv }}$. For the sake of expedience, we shall consider the bi-crystalline material where the grains 1 and 2 are separately by the grain boundary normal to $y$ direction and the platelet particles are regularly-arrayed periodically in $x$ direction. The length ( $y$-direction) and width (z-direction) of each platelet particle are assumed infinitely large compared with the thickness, $w_{\mathrm{p}}$ ( $x$-direction). A part of this system is schematically shown in Fig. 1 (a) where $L_{\mathrm{x}}$ corresponds to a distance between the platelet particles periodically arraying in $x$-direction. The interfacial energy between the particle and the grain 1 (grain 2) is denoted by $\sigma_{1}\left(\sigma_{2}\right)$. When the grain boundary moves the distance of $\Delta y$ in $+y$ direction, the resulting change of the total energy amounts to $2 \Delta y L_{z}\left(\sigma_{2}-\sigma_{1}\right)$. Hence, the energy change per the moved length, which corresponds to the inverse pinning force, is given by $2 L_{z}\left(\sigma_{2}-\sigma_{1}\right)$. The pressure exerting on the grain boundary, viz., the inverse pinning pressure is then obtained by dividing the inverse pinning force by the area of the grain boundary. When the particle thickness $w_{\mathrm{p}}$ is small compared with $L_{x}$, i.e., $f_{\mathrm{v}}$ is small, the area of the grain boundary can be approximated by $L_{z} L_{x}$. Then the pinning pressure is given by $\Delta P_{\text {inv }}=2\left(\sigma_{2}-\sigma_{1}\right) / L_{x}$. Since the volume fraction of the particle is $f_{\mathrm{v}}=w_{\mathrm{p}} / L_{x}$, one can obtain $\Delta P_{\text {inv }}=2\left(f_{\mathrm{v}} / w_{\mathrm{p}}\right)\left(\sigma_{2}-\sigma_{1}\right)$. The substitution of this relation into $\Delta G_{\text {inv }}=v_{\mathrm{m}} \Delta P_{\text {inv }}$ yields Eq. (1).

As discussed above, Eq. (1) is considered applicable to the systems with the small volume fraction of particles, because the area of the grain boundary is approximated by $L_{x} L_{z}$ in its derivation. This limitation can be readily removed as follow. When $w_{\mathrm{p}}$ is not sufficiently small, the area of the grain boundary is not $L_{x} L_{z}$ but $\left(L_{x}-w_{\mathrm{p}}\right) L_{z}$, as understood from Fig. 1(a). Hence, the inverse pinning pressure is given by $\Delta P_{i n v}=2 L_{z}\left(\sigma_{2}-\sigma_{1}\right) /\left(\left(L_{x}-w_{\mathrm{p}}\right) L_{z}\right)=2\left(\sigma_{1}-\sigma_{2}\right) /\left(L_{x}-w_{\mathrm{p}}\right)$. Using the relation $1 / L_{x}=f_{\mathrm{v}} / w_{\mathrm{p}}$, the pinning pressure is rewritten as $\Delta P_{i n v}=2 f_{\mathrm{v}}\left(\sigma_{2}-\sigma_{1}\right) /\left(w_{\mathrm{p}}\left(1-f_{\mathrm{v}}\right)\right)$. Hence, the driving force is given as

$$
\Delta G_{\mathrm{inv}}=v_{\mathrm{m}} \Delta P_{\mathrm{inv}}=\frac{2 v_{\mathrm{m}}}{w_{\mathrm{p}}}\left(\frac{f_{\mathrm{v}}}{1-f_{\mathrm{v}}}\right)\left(\sigma_{2}-\sigma_{1}\right) .
$$

In contrast to Eq. (1), $\Delta G_{\text {inv }}$ in Eq. (2) is not proportional to $f_{\mathrm{v}}$ as is. This correction becomes important when $f_{\mathrm{v}}$ is high. It is noted that Eq. (2) is reduced to Eq. (1) when $f_{\mathrm{v}}$ 
approaches null.

The most important fact understood from Eqs. (1) and (2) is that the driving force of the inverse pinning is proportional to the difference between the interfacial energies, i.e., $\Delta \sigma=\sigma_{2}-\sigma_{1}$. However, the maximum value actually exists in the driving force, above which the driving force is independent of $\Delta \sigma$. In order to make this point clear, we re-derive Eq. (2) on the basis of the curvature of the grain boundary. It is generally accepted that the migration velocity of a segment of the grain boundary is proportional to the pressure difference caused by its local curvature [2]. This pressure is given by $\sigma_{\mathrm{g}} / r$ with the local curvature radius of the boundary $r$ and the grain boundary energy $\sigma_{\mathrm{g}}$. The inverse pinning pressure $\Delta P_{\text {inv }}$ can be derived from this relation by taking into account the change of the curvature radius due to the particle-boundary interaction. In the inverse pinning phenomenon, the grain boundary is generally curved near the triple junction because of the energy balance between $\sigma_{1}, \sigma_{2}$ and $\sigma_{\mathrm{g}}$. The two-dimensional image of the inverse pinning process is depicted in Fig. 1(b) where $\theta$ is the angle between $y$-axis and the grain boundary at the triple junction. If the shape of the whole grain boundary can be approximated by the segment of a single circle, its curvature radius $r$ is described by $r=d /(2 \cos \theta)$. Here, $d$ is the thickness of the grain and it can be expressed in terms of $f_{\mathrm{v}}$ and $w_{\mathrm{p}}$ as $d=w_{\mathrm{p}}\left(1-f_{v}\right) / f_{\mathrm{v}}$. Hence, by substituting these relations into $\Delta P_{\text {inv }}=\sigma_{\mathrm{g}} / r$, the following equation for $\Delta G_{\text {inv }}$ is obtained,

$$
\Delta G_{\mathrm{inv}}=\frac{2 v_{m} \sigma_{g}}{w_{p}}\left(\frac{f_{v}}{1-f_{v}}\right) \cos \theta
$$

Here $\cos \theta$ is given as $\cos \theta=\left(\sigma_{2}-\sigma_{1}\right) / \sigma_{g}$, provided that the shape of the boundary at the triple junction takes the equilibrium shape according to Young's law. Therefore, Eq. (3) is completely equivalent to Eq. (2). However, the form of Eq. (3) explicitly presents an important fact that the upper limit exists in $\Delta G_{\text {inv }}$. The upper limit is realized when $\cos \theta=1$. This condition corresponds to the complete wetting of the interface between the grain 1 and the particle by the grain 2 .

Now, our focus is directed at the migration of the grain boundary due to the inverse pinning. The migration velocity of the grain boundary due to the inverse pinning $V_{\mathrm{g}}$ is given by using $\Delta P_{\text {inv }}$ as follows,

$$
\begin{gathered}
V_{\mathrm{g}}=m \Delta P_{\mathrm{inv}}=m \sigma_{\mathrm{g}} \frac{2}{w_{\mathrm{p}}}\left(\frac{f_{\mathrm{v}}}{1-f_{\mathrm{v}}}\right) \cos \theta \\
=2 \cos \theta V_{\mathrm{ref}}, \quad(4)
\end{gathered}
$$

where $m$ is the grain boundary mobility and

$$
V_{\mathrm{ref}}=m \sigma_{\mathrm{g}} \frac{1}{w_{\mathrm{p}}}\left(\frac{f_{\mathrm{v}}}{1-f_{\mathrm{v}}}\right)=\frac{m \sigma_{\mathrm{g}}}{d} .
$$


As already described above, $d\left(=w_{\mathrm{p}}\left(1-f_{\mathrm{v}}\right) / f_{\mathrm{v}}\right)$ is the thickness of the grain $2 . V_{\text {ref }}$ is called the reference velocity in this paper and it can be regarded as the velocity of the boundary in the system without the effect of the particle as will be discussed in section 4 . It is important to mention that the migration velocity of the grain boundary due to the inverse pinning is proportional to the reference velocity. The proportionality coefficient is entirely determined by the balance between the interfacial energies and the grain boundary energy. When the proportionality coefficient is maximum, viz., $\cos \theta=1, V_{\mathrm{g}}$ becomes twice as large as the reference velocity, which is the upper limit of the velocity due to the inverse pinning.

It should be pointed out that Eq. (2) was derived without the detailed consideration of the shape of the grain boundary. On the other hand, Eq. (3) was derived in the light of the curvature of the grain boundary. But the curvature radius of the grain boundary was approximated by a single value of $r$. In this paper, the validity of Eqs. (2)-(4) is examined by the phase-field simulations.

\section{Simulation method}

In this study, we utilized the multi-phase-field model [13-15]. Within this model, the grain structure is characterized by a set of phase-fields, $\left\{\phi_{i}(\mathbf{r}, t)\right\}$. The phase-field $\phi_{i}(\mathbf{r}, t)$ corresponds to a probability of finding a grain having a crystallographic orientation specified by the subscript $i(i=1,2,3, \ldots, N)$. Here, $\mathbf{r}$ is the spatial coordinate, $t$ is the time and $N$ is the total number of orientations considered in the simulation. $\phi_{i}$ varies from 0 to 1 and the grain boundary is described as the diffuse interface in which the orientation of grains gradually changes. The normalization condition $\Sigma_{i}^{N} \phi_{i}=1$ is satisfied in each spatial point. When we define a step function $s_{i}$ so that $s_{i}=1$ when $\phi_{i}>0$ and $s_{i}=0$ otherwise, the number of phases coexisting in a given spatial point $S(\mathbf{r}, \mathrm{t})$ is given by $S(\boldsymbol{r}, t)=\sum_{i=1}^{N} s_{i}(\boldsymbol{r}, t)$. The time evolution of $\phi_{i}$ is described by the following equation [14],

$$
\frac{\partial \phi_{i}}{\partial t}=\frac{2 M_{\phi}}{S} \sum_{j \neq i}^{N} s_{j} s_{i}\left(\frac{\partial F}{\partial \phi_{i}}-\frac{\partial F}{\partial \phi_{j}}\right),
$$

where $M_{\phi}$ is the phase-field mobility and

$$
\frac{\partial F}{\partial \phi_{i}}=\sum_{k \neq i}^{N}\left(\frac{\epsilon_{i k}^{2}}{2} \nabla^{2} \phi_{k}+\omega_{i k} \phi_{k}\right),
$$

where $\varepsilon_{i k}$ and $\omega_{i k}$ are the gradient energy coefficient and the height of the parabolic potential with a double obstacle in the $i k$ interface. These parameters are related to the 
interface thickness $W$ and the interfacial energy $\sigma_{i k}$ as given by $\epsilon_{i k}=2 \sqrt{W \sigma_{i k}} / \pi$ and $\omega_{i k}=4 \sigma_{i k} / W$ [14]. When the values of $\sigma_{i k}$ and $W$ are given, $\varepsilon_{i k}$ and $\omega_{i k}$ are uniquely fixed. $W$ was set to $6 \Delta x$ with a square grid spacing $\Delta x$ in all the simulations.

The time evolution equations (6) were discretized in a two-dimensional system based on standard second-order finite difference formulas with the square grid spacing $\Delta x$ and these equations were solved using a simple first-order Euler scheme. Figure 2 represents the schematic illustration of the computational domain. There are the grain 1 at the bottom of the system and the grains 2 in the upper part of the grain 1 . The second phase particles exist in the shape of platelet having a thickness of $w_{\mathrm{p}}$. The computational domain corresponds to the rectangular area surrounded by the dashed line. The mirror boundary condition was applied to each edge in $x$-direction, while the no-flux boundary condition was set along each edge in $y$-direction. The initial curvature radius of the grain boundaries is equivalent to half of the system size of $x$-direction.

We have investigated the grain boundary migration in the system with the particle which is inert, viz., immobile. This process was simulated by solving the time evolution equations (6) for all the phase-fields except for the phase-field for the particle. In order to analyze the inverse pinning effect systematically, we focused on the processes with the different interfacial energies between the matrix and the particle. The interfacial energy between the grain 1 (grain 2) and particle is denoted by $\sigma_{1}\left(\sigma_{2}\right)$ and the grain boundary energy is described by $\sigma_{\mathrm{g}}$. The difference $\Delta \sigma=\sigma_{2}-\sigma_{1}$ was changed from 0 to $1.6 \mathrm{~J} \mathrm{~m}^{-2}$ and three values of $\sigma_{\mathrm{g}}=0.4,0.79$ and $1.2 \mathrm{~J} \mathrm{~m}^{-2}$ were employed. The phase-field mobility was set to $M_{\phi}=5.16 \times 10^{-3} \mathrm{~m}^{3} \mathrm{~J}^{-1} \mathrm{~s}^{-1}$. Although we do not specify the materials and the condition of the grain growth such as the temperature, a set of these input parameters reproduces the kinetics which is similar to the austenite grain growth of low carbon steels $(\sim 0.2$ mass $\%$ carbon steel) at a relatively high temperature $(\sim 1740$ K) [15]. In addition, the volume fraction and the particle thickness were chosen to be $f_{v}$ $=0-0.72, w_{\mathrm{p}}=0-36 \mu \mathrm{m}$. The square grid spacing $\Delta x$ was set to $1.0 \mu \mathrm{m}$, unless otherwise stated. The time step $\Delta t$ was given by $\Delta t=\Delta x^{2} /\left(5 M_{\phi} \varepsilon_{\mathrm{a}}{ }^{2}\right)$ where $\varepsilon_{\mathrm{a}}$ is the maximum value among the different $\varepsilon_{i k}$ associated with the different interfacial (grain boundary) energies. The simulations were conducted until the system reaches the steady state. The system size in $x$-direction was changed according to the given values of $d$ and $w_{\mathrm{p}}$, while the system size in $y$-direction was changed from $100 \mu \mathrm{m}$ to $500 \mu \mathrm{m}$ in order for the system to reach the steady state in all the growth conditions within reasonable computational times.

\section{Results and discussion}


We first investigate the grain boundary migration in the system without the second phase particle, from which the velocity equivalent to $V_{\text {ref }}$ in Eq. (5) is calculated. Figure 3 represents the time evolution of the microstructure calculated for $d=100 \mu \mathrm{m}$ and $\sigma_{\mathrm{g}}=0.79 \mathrm{~J} \mathrm{~m}^{-2}$. The grain boundary between the grains 1 and 2 moves upward with time. The temporal changes of the moved distance of the rearmost segment of the grain boundary are shown in Fig. 4(a) for different values of $d$. In all the cases, the velocity takes almost a constant after $t=0.15 \mathrm{~s}$. Figure 4(b) shows the moving velocity during the steady state (circle plots) obtained for the different values of $d$.

The Allen-Cahn type Eq. (6) exactly reproduces the well-known relationship for the curvature-driven motion of the boundary as given by $v=-m \sigma_{\mathrm{g}} / r$ where $v$ is the normal velocity of the grain boundary. Furthermore, the present phase-field model exactly reproduces von Neumann-Mullins law of two-dimensional grain growth kinetics [14]. This indicates that the kinetics of triple junctions does not directly affect the grain growth and the contact angles at the triple junction always comply with the equilibrium relationship, i.e., Young's law. The angle $\theta_{i}$ shown in Fig. 3(b) indicate the angle between $y$-axis and the grain boundary at the triple junction. Using $\theta_{i}, r$ is given by $r=$ $d /\left(2 \cos \theta_{i}\right)$ with the grain thickness $d$. In Figs. 3(b) and (c), the contact angle of the grain boundaries at the triple junction is $120^{\circ}$ and $\theta_{i}$ is accordingly $60^{\circ}$ in all the cases. Hence, the moving velocity of the boundary $v$ is given by $v=m \sigma_{\mathrm{g}} / d$. This is equivalent to the velocity $V_{\text {ref }}$ defined in Eq. (5). The calculated result of Eq. (5) is indicated by the solid line in Fig. 4(b). The results of phase-field simulation are completely in accord with Eq. (5).

The inverse pinning effect is discussed. Figure 5 shows the snapshots of the microstructure during the processes with the platelet particle, obtained from the phase-field simulations for $d=88 \mu \mathrm{m}, w_{\mathrm{p}}=12 \mu \mathrm{m}, \sigma_{\mathrm{g}}=\sigma_{1}=0.79 \mathrm{~J} \mathrm{~m}^{-2}$ and the different values of $\sigma_{2}$ and thus different values of $\Delta \sigma\left(=\sigma_{2}-\sigma_{1}\right)$. In the case of $\Delta \sigma=0$ (Fig. 5(a)), the grain boundary is completely pinned by the platelet particle. Namely the usual pinning effect is observed for $\Delta \sigma=0$. The complete stop of the grain boundary can be understood from the fact that the shape of the grain boundary is straight, viz., the curvature is null. When $\Delta \sigma$ increases (Figs. 5(b) and (c)), the grain boundary is curved. In these cases, the grain boundary moves with the time. When $\Delta \sigma=1.6 \mathrm{~J} \mathrm{~m}^{-2}$ (Fig. 5(d)), the grain boundary does not directly touch the particle, being more curved. In this case, the interface between the particle and grain 2 is completely wetted by the grain 1 . It moves with higher velocity than those in the former cases of $\Delta \sigma=0.16$ and $0.47 \mathrm{~J} \mathrm{~m}^{-2}$. The dependences of the moving velocity $V_{\mathrm{g}}$ on $\Delta \sigma$ were calculated for different sets of $\sigma_{\mathrm{g}}, \sigma_{1}$ and $\sigma_{2}$. The results are shown in Fig. 6. In each set of data, the values of $\sigma_{\mathrm{g}}$ and 
$\sigma_{1}$ are fixed and $\sigma_{2}$ is varied. In all the cases, $V_{\mathrm{g}}$ increases with increase in $\Delta \sigma$. When the particle does not exist, the moving velocity $V_{\text {ref }}$ for $d=88 \mu \mathrm{m}$ corresponds to about 220 $\mu \mathrm{m} \mathrm{s}^{-1}$ as shown in Fig. 4(b). Hence, the grain boundary migration is accelerated by the particle in all the cases when $\Delta \sigma$ is sufficiently high.

It is noted in Fig. 6 that $V_{\mathrm{g}}$ shows the upper limit for each value of $\sigma_{\mathrm{g}}$ when $\Delta \sigma$ takes high values. For instance, when $\sigma_{\mathrm{g}}=\sigma_{1}=0.79 \mathrm{~J} \mathrm{~m}^{-2}, V_{\mathrm{g}}$ takes almost a constant value of about $700 \mu \mathrm{m} \mathrm{s}^{-1}$ for $\Delta \sigma>0.8 \mathrm{~J} \mathrm{~m}^{-2}$. According to Eq. (4), this upper limit is given by $2 V_{\text {ref. }}$ In order to see the validity of Eq. (4), $V_{\mathrm{g}}$ is divided by $V_{\text {ref }}$ calculated for $d=88 \mu \mathrm{m}$ and its normalized velocity is plotted against $\Delta \sigma / \sigma_{\mathrm{g}}(=\cos \theta)$ in Fig. 7 where the solid line indicate the relation $V_{\mathrm{g}} / V_{\text {ref }}=2 \cos \theta$, viz., Eq. (4). Regardless of the values of $\sigma_{\mathrm{g}}$ and $\sigma_{1}$, all data merge into the single curve of $V_{\mathrm{g}} / V_{\text {ref }}=2 \cos \theta$. When $\Delta \sigma / \sigma_{\mathrm{g}}$ is less than 0.5 , this theoretical relation is in complete agreement with the results of the phase-field simulations. On the other hand, for $\Delta \sigma / \sigma_{\mathrm{g}}>0.5$, the results of the simulations deviate from this theoretical relation. However, this does not indicate the failure of Eq. (4). This is due to the numerical error in the phase-field simulations. When $\Delta \sigma / \sigma_{\mathrm{g}}>0.5$, the contact angle of the boundaries at the triple junction $\theta$ becomes very small. We found it quite difficult to precisely describe such small angles in the present phase-field simulations. This error can be gradually reduced by decreasing $\Delta x$. The dependence of the results of phase-field simulation on $\Delta x$ is shown in Fig. 8. The data of the phase-field simulations gradually approach the solid line of Eq. (4) as $\Delta x$ decreases. Hence, the present simulations show the validity of Eq. (4) regarding the dependence of $V_{\mathrm{g}}$ on $\Delta \sigma / \sigma_{\mathrm{g}}$. It is noted that the convergence is not fast in Fig. 8 and therefore we decided to use $\Delta x=1.0 \mu \mathrm{m}$ in the light of the computational burden and the accuracy.

Figure 9 represents the dependence of $V_{\mathrm{g}}$ on $\Delta \sigma / \sigma_{\mathrm{g}}$ calculated for $w_{\mathrm{p}}=12 \mu \mathrm{m}$, $\sigma_{\mathrm{g}}=\sigma_{1}=0.79 \mathrm{~J} \mathrm{~m}^{-2}$ and the different value of $f_{\mathrm{v}}$. The solid lines are the results of Eq. (4) for the different value of $f_{\mathrm{v}}$. Although there are the differences between Eq. (4) and the simulation results for high values of $\Delta \sigma / \sigma_{\mathrm{g}}$ due to the numerical error described above, the results of Eq. (4) coincide well with those of phase-field simulations for each value of $f_{\mathrm{v}}$.

As described in section 2, the essential difference between Eqs. (1) and (2) is in the dependence of $\Delta G_{\text {inv }}$ (thus $V_{\mathrm{g}}$ ) on $f_{\mathrm{v}}$ and $w_{\mathrm{p}}$. According to Eq. (1), $V_{\mathrm{g}}$ is determined only by the ratio of $f_{\mathrm{v}} / w_{\mathrm{p}}$ for a given value of $\Delta \sigma$. In other words, it should be independent of $w_{\mathrm{p}}$ or $f_{\mathrm{v}}$ as long as the ratio $f_{\mathrm{v}} / w_{\mathrm{p}}$ is a constant. The dependences of $V_{\mathrm{g}}$ on $w_{\mathrm{p}}$ for fixed values of $w_{\mathrm{p}} / f_{\mathrm{v}}$ are shown in Fig. 10 where the plots and the solid lines are the results of the phase-field simulations and Eq. (4), respectively. $\Delta \sigma$ was set to $\Delta \sigma=$ 
0.5. $V_{\mathrm{g}}$ clearly depends on $w_{\mathrm{p}}$ even though $f_{\mathrm{v}} / w_{\mathrm{p}}$ is fixed, which indicate that Eq.(1) is not applicable to the system with high volume fraction. On the other hand, the simulation results agree well with the results of Eq. (4) and it shows the validity of Eqs. (2) and (3). The correction as given by Eqs. (2) and (3) becomes important when $f_{\mathrm{v}}$ exceeds 0.1 .

As demonstrated above, the present phase-field simulations demonstrated the validity of Eqs. (2)-(4). It is important to use Eq. (2) or (3) instead of Eq. (1) when the volume fraction of particles is relatively high. In addition, there exists the maximum velocity of grain boundary migrating due to the inverse pinning force, which is determined not by $\Delta \sigma=\sigma_{2}-\sigma_{1}$ but by $m$ and $\sigma_{\mathrm{g}}$ for given volume fraction and thickness of the particles.

\section{Summary}

In this study, the theoretical and numerical investigations were carried out into effects of the inverse pinning of platelet particles on the grain boundary migration. The theoretical expression for the driving force of the inverse pinning of platelet particles proposed in the early report [11] was first derived and it was discussed that this expression is valid for the system with small volume fraction. In this paper, we derived the driving force of the inverse pinning which is free from the limitation in the volume fraction. In addition, by considering the curvature-driven motion of the boundary, we showed that the velocity of the grain boundary reaches at its maximum in the complete wetting condition. These theoretical considerations were then examined by the phase-field simulations for the grain growth. The validity of our discussions was successfully demonstrated.

The focus in this paper was placed on the very simplified system where the platelet particles having infinitely large length and width are regularly arrayed along $x$-direction. In reality, however, the precipitates have finite length and width and they are not regularly arrayed. The development for the theory of inverse pinning for more general cases remains to be tacked in a future work. 


\section{References}

[1] C.S. Smith, Trans. AIME. 175 (1948) 15-51 .

[2] M. Hillert, Acta Metall. 13 (1965) 227-238.

[3] T. Gladman, Proc. R. Soc. Lond. A294 (1966) 298-309.

[4] N. Louat, Acta Metall. 30 (1982) 1291-1294.

[5] E. Nes, N. Ryum and O. Hunderi, Acta Metal. 33 (1985) 11-22.

[6] P.R. Rios, Acta Metall. 35 (1987) 2805-2814.

[7] P.A. Manohar, M. Ferry and T. Chandra, ISIJ Int. 38 (1998) 913-924.

[8] M.A. Miodownik, Scripta Mater. 54 (2006) 993-997.

[9] A. Harun, E.A. Holm, M.P. Clode, M.A. Miodownik, Acta Mater. 54 (2006) 3261-3272.

[10] N. Moelans, B. Blanpain, P. Wollants, Acta Mater. 55 (2007) 2173-2182.

[11] T. Nishizawa, ISIJ Int. 40 (2000) 1269-1274.

[12] Y. Suwa, Y. Saito, H. Onodera, Acta Mater. 55 (2007) 6881-6891.

[13] I. Steinbach, F. Pezzola, Physica D 134 (1999) 385-393.

[14] S.G. Kim, D.I. Kim, W.T. Kim, Y.B. Park, Phys. Rev. E 74 (2006) 061605-1-14.

[15] M. Ohno, S. Tsuchiya, K. Matsuura, Acta Mater. 59 (2011) 5700-5709. 
(a)

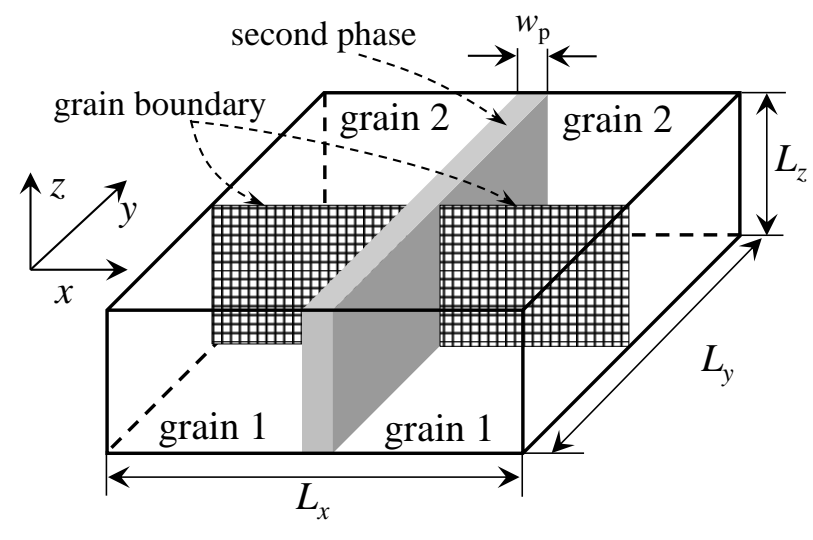

(b)

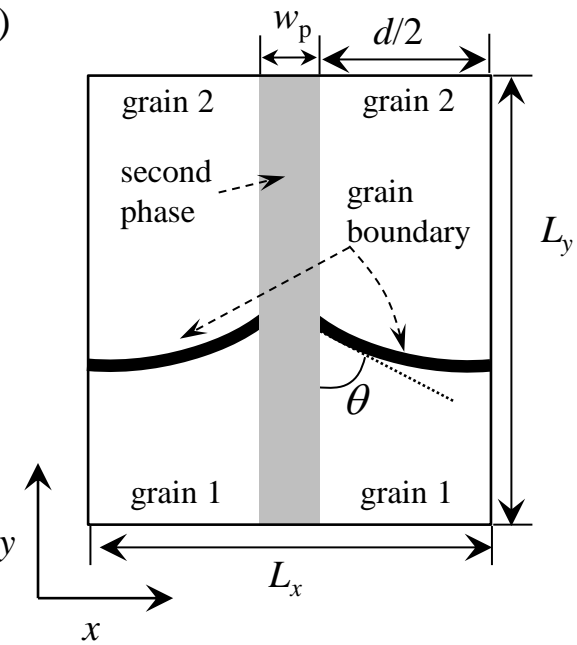

Fig. 1. (a) three-dimensional and (b) two-dimensional illustrations of the inverse pinning process. 


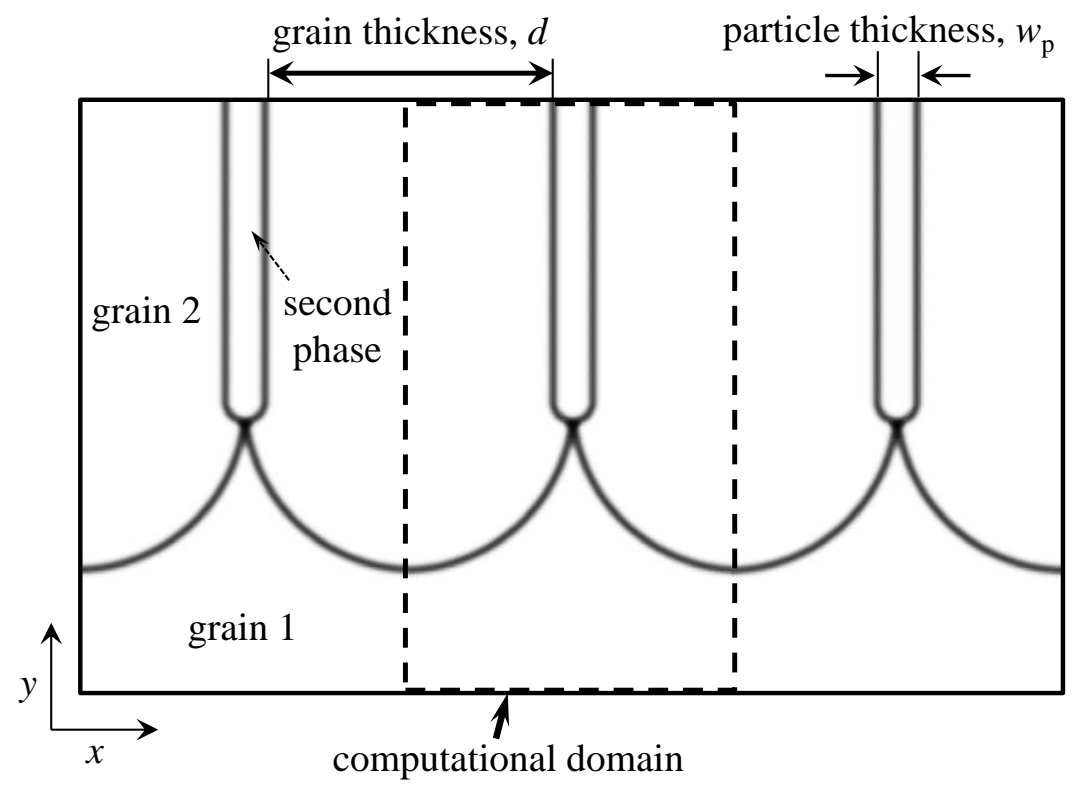

Fig. 2. Schematic illustration of computational domain (surrounded by dashed line). 
(a) $t=0.0 \mathrm{~s}$

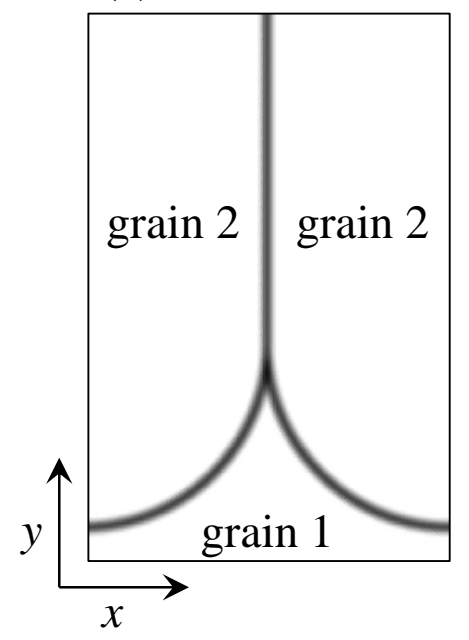

(b) $t=0.1 \mathrm{~s}$

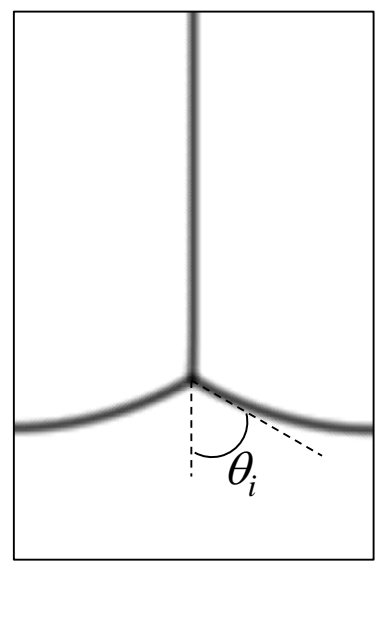

(c) $t=0.4 \mathrm{~s}$

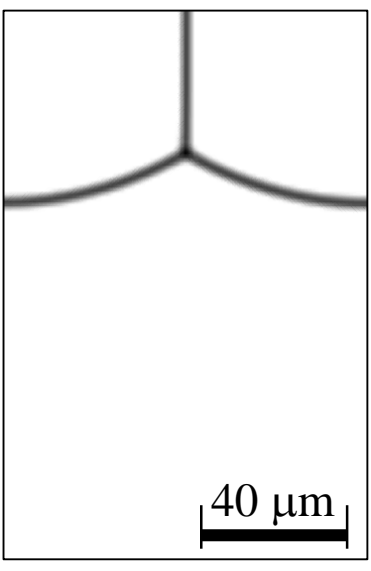

Fig. 3. Snapshots of microstructures during the grain growth without the second phase particles at different times. Thick lines indicate the grain boundaries in each figure. 
(a)

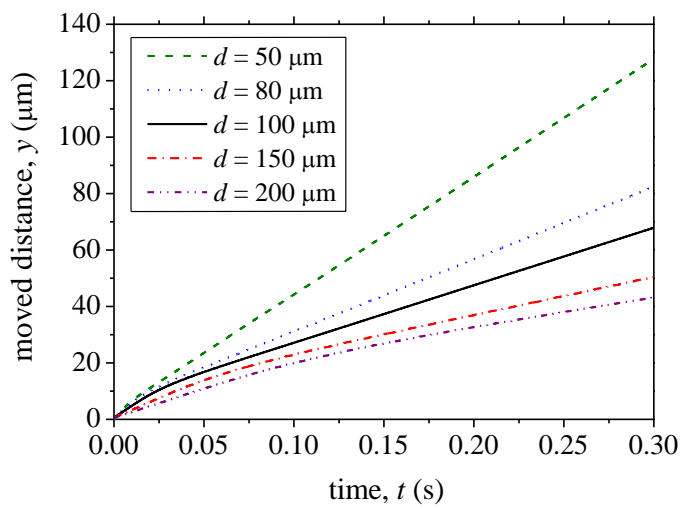

(b)

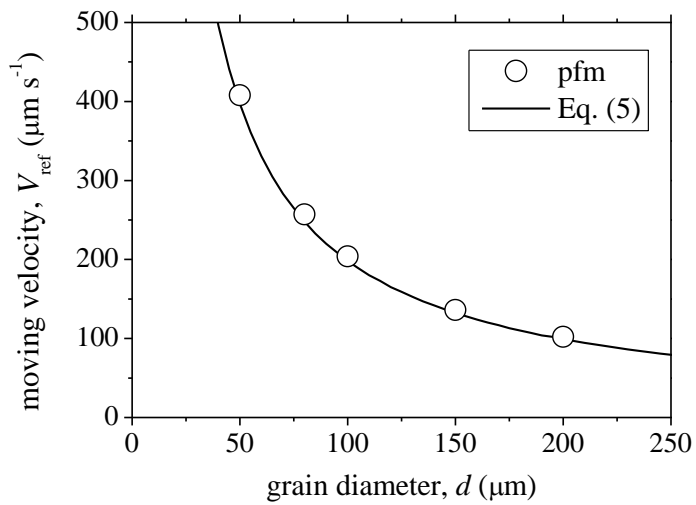

Fig. 4. (a) Time change of moved distance for different value of $d$ in the system without the second phase. (b) Dependence of moving velocity during the steady state $V_{\text {ref }}$ on $d$. The plots are the results of phase-field simulations, while the solid line indicates the result of Eq. (5). 
(a) $\Delta \sigma=0.0 \mathrm{~J} \mathrm{~m}^{2}$

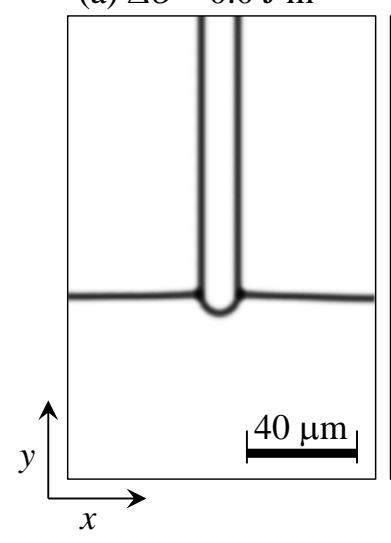

(b) $\Delta \sigma=0.16 \mathrm{~J} \mathrm{~m}^{2}$

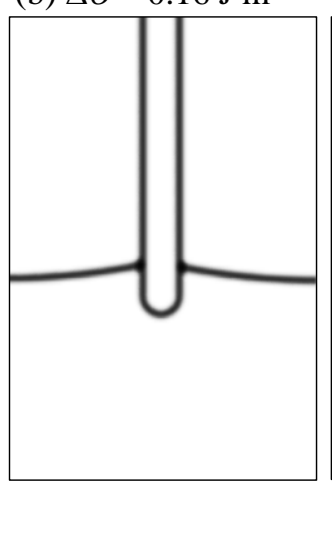

(c) $\Delta \sigma=0.47 \mathrm{~J} \mathrm{~m}^{2}$

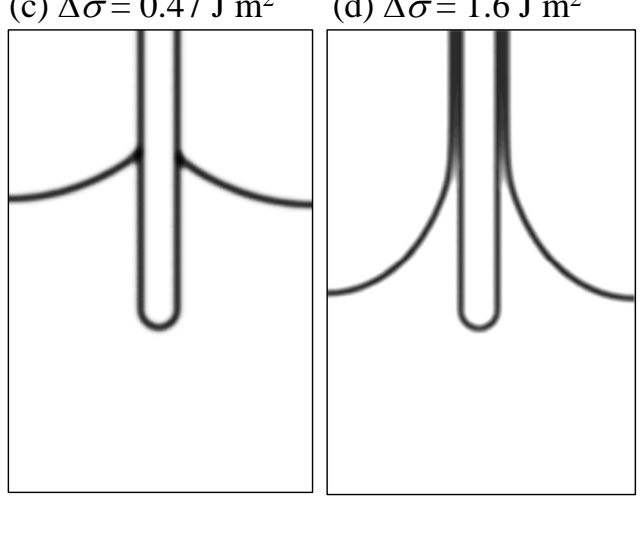

Fig. 5. Snapshots of microstructure with the second phase particle at (a)-(c) $t=0.3$ and (d) $t=0.15 \mathrm{~s}$ simulated for $d=88 \mu \mathrm{m}, w_{\mathrm{p}}=12 \mu \mathrm{m}, \sigma_{\mathrm{g}}=\sigma_{1}=0.79 \mathrm{~J} \mathrm{~m}^{-2}$ and the different values of $\Delta \sigma=\sigma_{2}-\sigma_{1}$. 


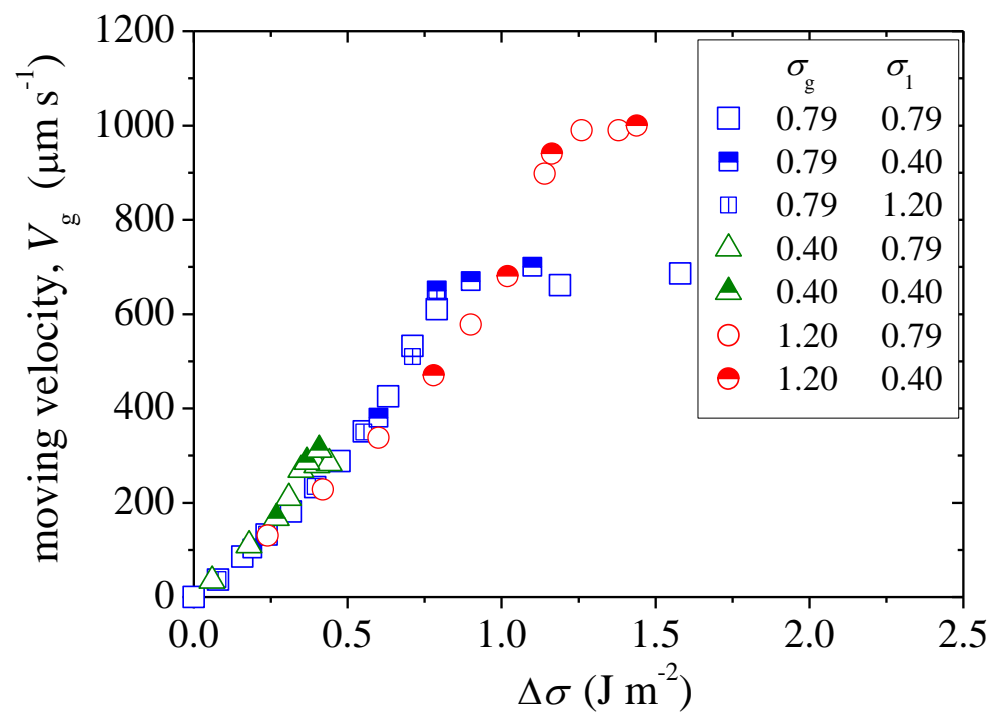

Fig. 6. Dependences of $V_{\mathrm{g}}$ on $\Delta \sigma$ calculated for $d=88 \mu \mathrm{m}, w_{\mathrm{p}}=12 \mu \mathrm{m}$ and different sets of $\sigma_{\mathrm{g}}, \sigma_{1}$ and $\sigma_{2}$. In the legend, the values of interfacial energies are denoted in the unit of $\mathrm{J} \mathrm{m}^{-2}$. 


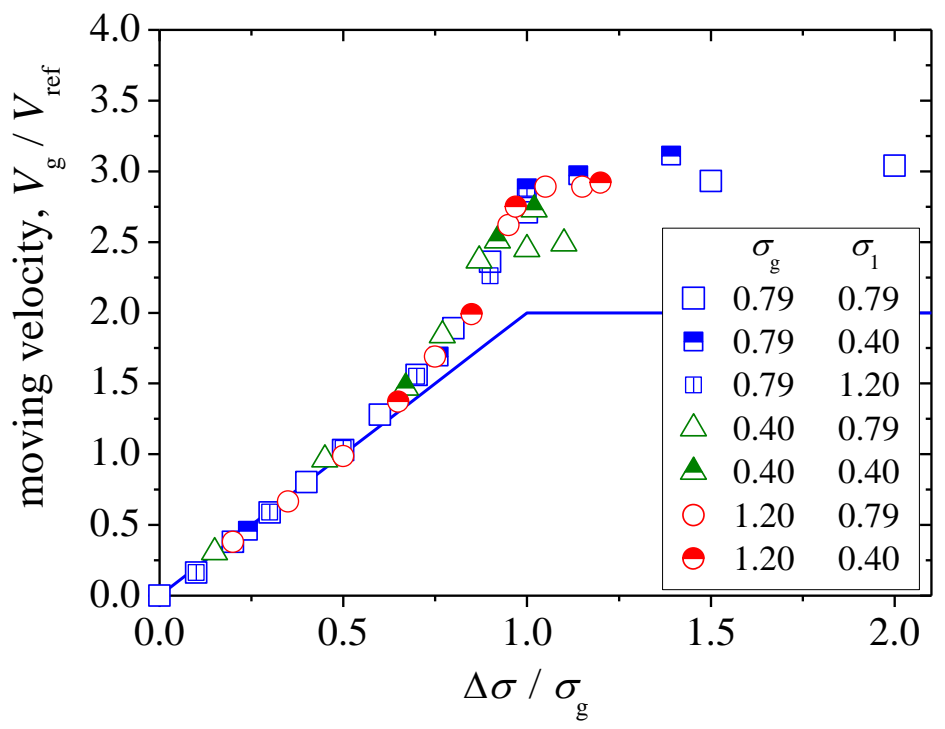

Fig. 7. Dependences of $V_{\mathrm{g}} / V_{\text {ref }}$ on $\Delta \sigma / \sigma_{\mathrm{g}}$ calculated for $\Delta x=1 \mu \mathrm{m}, d=88 \mu \mathrm{m}, w_{\mathrm{p}}=12$ $\mu \mathrm{m}$ and different sets of $\sigma_{\mathrm{g}}, \sigma_{1}$ and $\sigma_{2}$. In the legend, the values of interfacial energies are denoted in the unit of $\mathrm{J} \mathrm{m}^{-2}$. The solid line indicates the result of Eq. (4). 


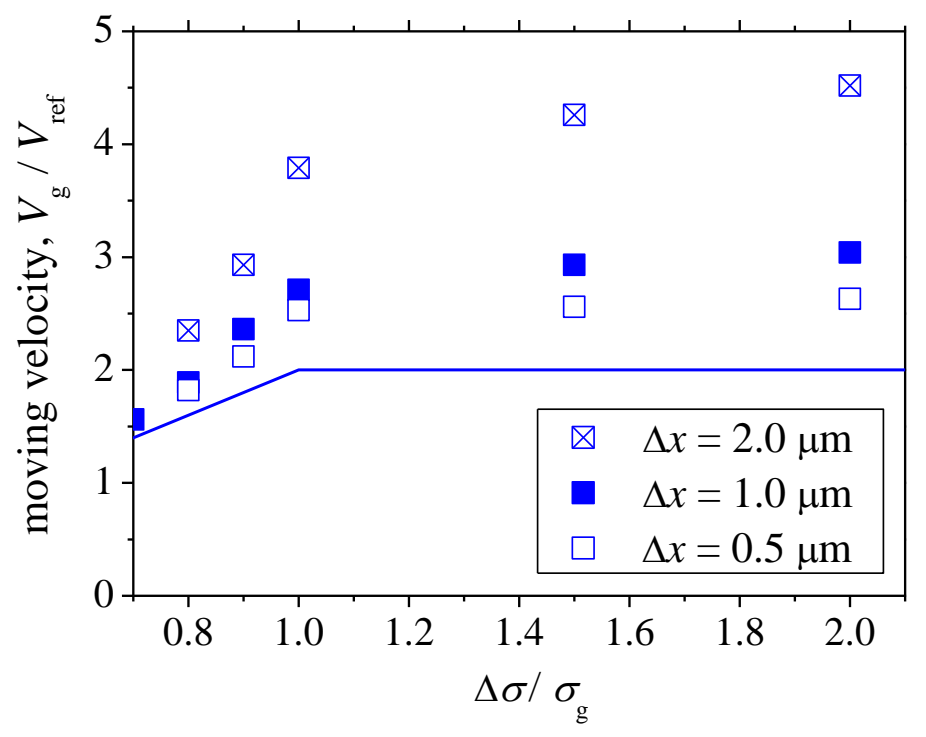

Fig. 8. Grid size dependence of $V_{\mathrm{g}} / V_{\text {ref }}$ calculated for $\sigma_{\mathrm{g}}=\sigma_{1}=0.79 \mathrm{~J} \mathrm{~m} \mathrm{~m}^{-2}$. The solid line indicates the result of Eq. (4). 


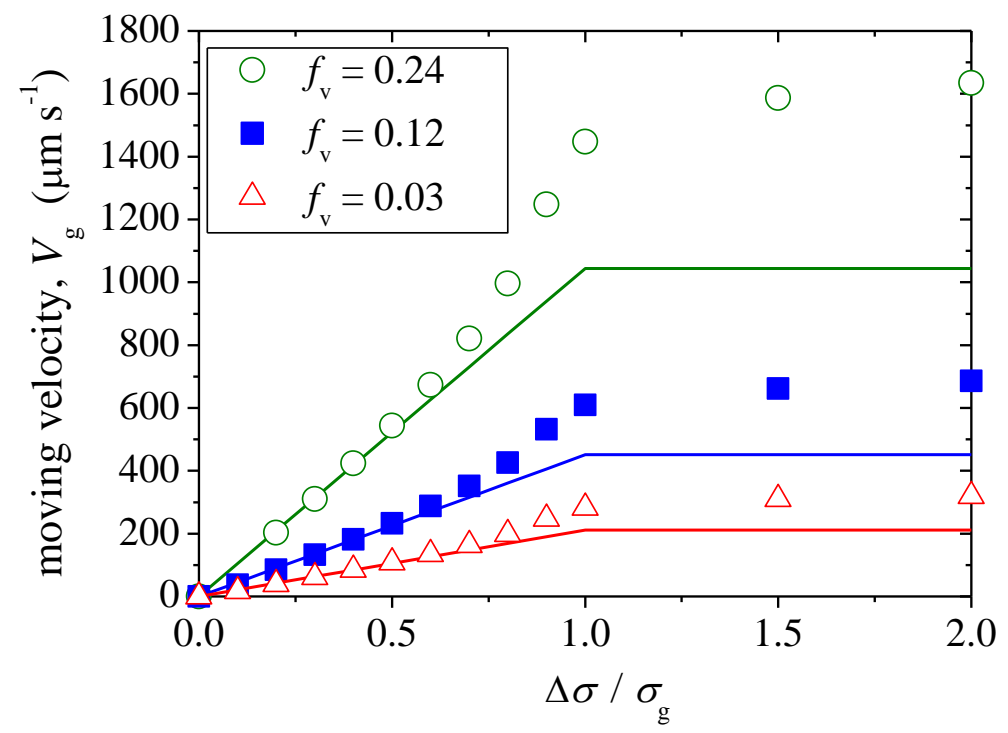

Fig. 9. $V_{\mathrm{g}}$ with respect to $\Delta \sigma / \sigma_{\mathrm{g}}$ calculated for $w_{\mathrm{p}}=12 \mu \mathrm{m}, \sigma_{\mathrm{g}}=\sigma_{1}=0.79 \mathrm{~J} \mathrm{~m}^{-2}$ and the different values of $f_{\mathrm{v}}$. Three lines are the results of Eq. (4) for three values of $f_{\mathrm{v}}$. 


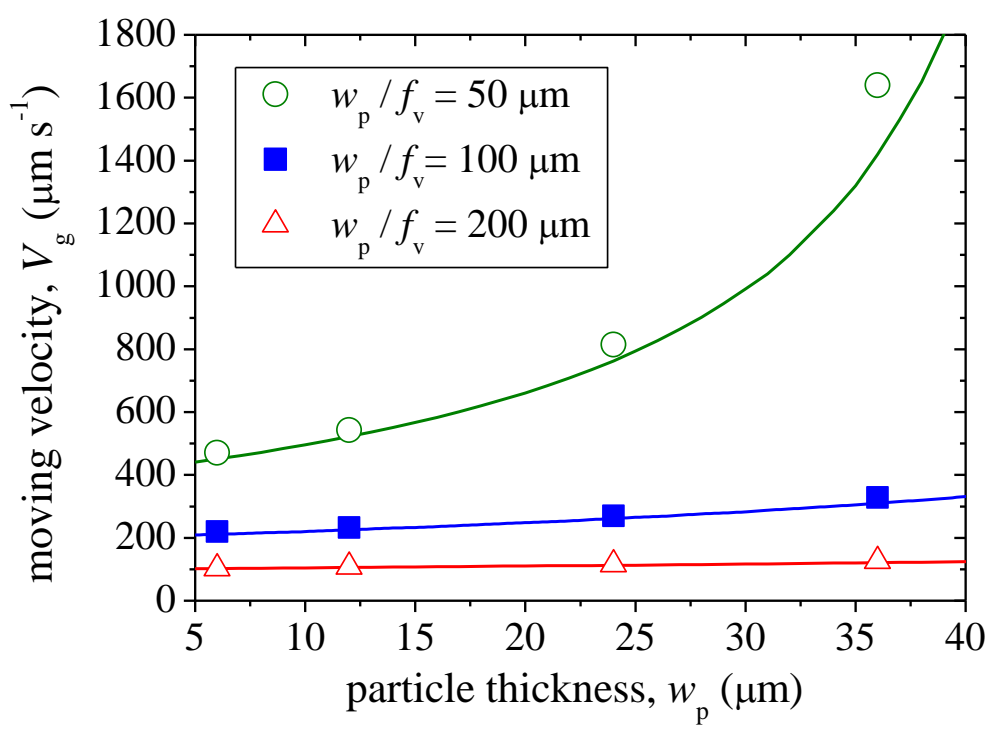

Fig. 10. Dependence of $V_{\mathrm{g}}$ on $w_{\mathrm{p}}$ calculated for $\Delta \sigma / \sigma_{\mathrm{g}}=0.5$ and the different values of $w_{\mathrm{p}} / f_{\mathrm{v}}$. The lines are the results of Eq. (4) for three values of $w_{\mathrm{p}} / f_{\mathrm{v}}$. 